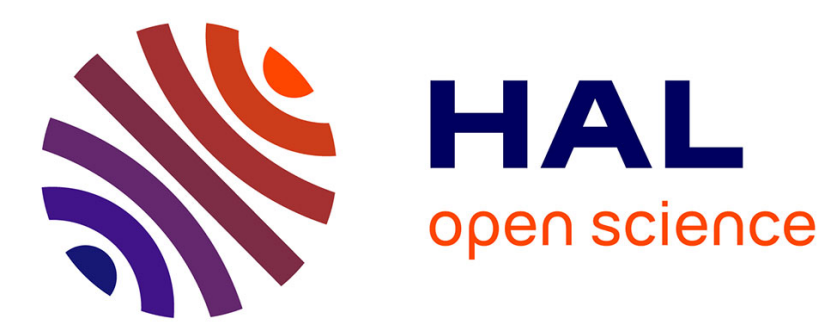

\title{
Enclaves et enclavement dans le Ferghana post-soviétique
}

\author{
Julien Thorez
}

\section{To cite this version:}

Julien Thorez. Enclaves et enclavement dans le Ferghana post-soviétique. Cahiers d'études sur la Méditerranée orientale et le monde turco-iranien, 2003, 35, pp. 28-39. halshs-01375995

\section{HAL Id: halshs-01375995 https://shs.hal.science/halshs-01375995}

Submitted on 4 Oct 2016

HAL is a multi-disciplinary open access archive for the deposit and dissemination of scientific research documents, whether they are published or not. The documents may come from teaching and research institutions in France or abroad, or from public or private research centers.
L'archive ouverte pluridisciplinaire HAL, est destinée au dépôt et à la diffusion de documents scientifiques de niveau recherche, publiés ou non, émanant des établissements d'enseignement et de recherche français ou étrangers, des laboratoires publics ou privés. 


\title{
Cahiers d'études sur la Méditerranée orientale et le monde turco-iranien (CEMOTI), $\mathrm{n}^{\circ}$ 35, 2003, pp. 28-39
}

\section{Enclaves et enclavement dans le Ferghana post-soviétique}

\author{
Julien Thorez \\ Agrégé de géographie
}

ATER à l'université de Nantes

\begin{abstract}
Résumé : Au cœur de l'Asie centrale post-soviétique, le bassin du Ferghana, compartiment densément peuplé, est partagé entre l'Ouzbékistan, le Kirghizstan et le Tadjikistan. Le tracé complexe des frontières est le produit du découpage soviétique de l'Asie centrale. La région comprend ainsi une dizaine d'enclaves. Depuis 1991, la disparition de l'URSSet l'indépendance des républiques transforment le statut des frontières qui, en raison des politiques nationales post-soviétiques, notamment en Ouzbékistan, deviennent difficilement franchissables. Les enclaves de Sokh, Chakhimardan et Voroukh connaissent des évolutions différentes dans ce nouveau contexte. La principale conséquence des transformations post-soviétiques est la généralisation de l'enclavement en particulier au sud du Kirghizstan et au nord du Tadjikistan. Mais, surtout, dix ans d'indépendance abutissent à la fragmentation de l'espace régional et à l'apparition de trois Ferghana distincts.
\end{abstract}

Au cœur de l'Asie centrale, le Ferghana est un vaste bassin intra-montagnard, situé entre les extrémités occidentales du Tian-Chan ${ }^{1}$. Dans ses dimensions maximales, la dépression s'étend sur $300 \mathrm{~km}$ d'ouest en est et sur $170 \mathrm{~km} \mathrm{du}$ nord au sud, pour une superficie d'environ $22000 \mathrm{~km}^{2}{ }^{2}$. Oasis ancienne, ce bassin constitue l'un des principaux foyers de peuplement et l'une des régions agricoles les plus riches d'Asie centrale. Sa population composite comprend plus de 10 millions d'habitants - environ 2,5 millions au Kirghizstan, près de 7 millions en Ouzbékistan et autour de 1,5 millions au Tadjikistan. La densité y est donc élevée, l'oblast' d'Andijan étant même la région administrative la plus densément peuplée de l'ancienne Union soviétique avec une densité supérieure à 500 hab. $/ \mathrm{km}^{2}$.

À l'époque soviétique, le Ferghana formait une région unie caractérisée par un système de peuplement et une intégration économique qui transcendaient les limites territoriales malgré un tracé des frontières complexe ${ }^{3}$. Depuis 1991, la transformation postsoviétique redessine la géographie du Ferghana et de l'Asie centrale plus généralement. L'indépendance et les «transitions » font évoluer le système spatial ferghanien dans ses composantes réticulaires et aréolaires d'autant plus vigoureusement que le bassin est fragmenté par les subdivisions administratives de trois États différents.

\footnotetext{
${ }^{1}$ Les monts de Kurama et la chaîne de Tchatkal au nord, la chaîne du Ferghana à l'est, et la chaîne de l'Alaï et les monts du Turkestan au sud encadrent le bassin du Ferghana.

${ }^{2}$ Srednjaja Azija (serija «Prirodnye uslovija i estestvennye resursy SSSR ») [L’Asie moyenne, (série : «Les conditions naturelles et les ressources naturelles de l'URSS »)], Moscou, Nauka, 1968, p. 356.

${ }^{3}$ Saliev, A. S., Faktory i tendentsii razvitija sovremennogo rasselenija v respublikah Srednej Azii [Facteurs et tendances du développement du peuplement contemporain dans les républiques d'Asie moyenne], thèse de doctorat d'État, Tachkent, 1986, pp. 225-230.
} 


\section{Un découpage frontalier complexe hérité de la période soviétique.}

La complexité du tracé des frontières dans le Ferghana est remarquable. Tout d'abord, bien que de taille relativement modeste à l'échelle de l'Asie moyenne, le bassin forme un ensemble naturel qui est divisé en trois territoires républicains distincts. L'Ouzbékistan contrôle la plaine centrale et un étroit corridor au nord-ouest qui relie les oblast' d'Andijan, de Ferghana et de Namangan au reste du territoire national. L'ouverture occidentale, la «porte de Khodjent », constitue le nord du territoire tadjikistanais, tandis que les piémonts périphériques et les pourtours montagneux sont situés au Kirghizstan. De plus, le dessin même des frontières se révèle très compliqué dans le détail, les rentrants et les saillants dessinant une carte complexe. La longueur des frontières par rapport à la surface de ce foyer de peuplement ancien est donc importante. Par ailleurs, le Ferghana comprend une dizaine d'enclaves, définie comme « territoire inclus dans un espace placé sous une autorité qui lui est étrangère ${ }^{4}$. Ces inclusions de superficie réduite accentuent la complexité frontalière du Ferghana. Situées au Kirghizstan mais rattachées à l'Ouzbékistan et au Tadjikistan, Sokh, Chakhimardan et Voroukh sont les principales (voir carte : «les enclaves du Ferghana»). Cette densité exceptionnelle d'enclaves appartenant désormais à trois états indépendants constitue une caractéristique et une originalité du Ferghana.

Tracées entre 1924 et 1929 dans leur ensemble, les frontières du Ferghana sont un héritage soviétique majeur tout comme l'existence même des républiques centre-asiatiques. Ce choix soviétique de créer les républiques centre-asiatiques en divisant le Turkestan et le dessin de leurs frontières sont des legs soviétiques controversés, par des auteurs locaux ${ }^{5}$ comme par des auteurs occidentaux ${ }^{6}$. Les frontières du Ferghana si complexes soient-elles ne sont pas arbitraires ni plus artificielles qu'ailleurs, mais le produit d'une période et de la confrontation de différents critères dans le cadre d'une lutte politique entre les dirigeants soviétiques centreasiatiques $^{7}$. Ainsi, les Kirghizes de l'oblast' montagnard de Kirghizie qui devint RSSA en 1926 se sont affrontés avec les Ouzbeks gouvernant la république du Turkestan puis la RSS d'Ouzbékistan à propos de la localisation des frontières. Entre 1924 et 1927, le Kirghizstan revendiqua la possession de nombreux territoires de la plaine ferghanienne centrale (au sudest d'Andijan, au sud de Ferghana, vers Isfara et au sud de Namangan notamment) et les enclaves de Chakhimardan, Sokh et Voroukh. Pour sa part, l'Ouzbékistan affirmait ses prétentions sur la ville d'Och et ses alentours de même que sur la région de Djalal-Abad.

Par delà les rapports de force politiques, le tracé des frontières dans le Ferghana est également fondé sur des critères de nature variée : linguistique, économique, symbolique. La langue est ainsi un élément de différenciation sur lequel les Soviétiques se sont appuyés sans qu'il ne soit exclusif ni appliqué systématiquement. Si effectivement le cœur de la cuvette ferghanienne contenait une population majoritairement ouzbèke au moment du découpage des

\footnotetext{
${ }^{4}$ Steck, B., Enclavement-désenclavement, une problématique du développement, HDR, Université du Havre, 2000, tome 2, p 130.

${ }^{5}$ Cette attitude est notamment celle d'une partie de l'intelligentsia tadjike qui n'accepte pas la «perte» de Samarcande et de Boukhara (voir par exemple: Masov, R., Istorija Topornogo razdelenija [Histoire d'un découpage à la hache], Douchanbe, 1991, Irfon, 192 p.).

${ }^{6} \mathrm{D}$. Balland considère ainsi que le principe même de découpage de l'Asie centrale en plusieurs républiques ne pouvait être satisfaisant tandis qu'O. Roy estime que les républiques ont été créés à l'encontre de toute rationalité géographique, économique ou ethnique pour empêcher qu'elles soient viables (Roy, O., La nouvelle Asie centrale ou la fabrication des nations, Paris, 1997, Editions du Seuil, 331 p.).

${ }^{7}$ Koïchiev, A., Nacional'no-territorial'noe razmeževanije v ferganskoj doline (1924-27), [La délimitation des territoires nationaux dans la vallée du Ferghana (1924-27)], Bichkek, 2001, 120 p.
} 
frontières, l'enclave de Sokh, qui fut rattachée à l'Ouzbékistan était peuplée de Tadjiks ! Sur le plan économique, le partage territorial assure à chacune des républiques des terroirs agricoles irrigués de piémont, c'est-à-dire l'existence d'une agriculture riche, alors même que le Kirghizstan et le Tadjikistan sont surtout montagnards. Enfin, chacune des républiques détient sur son territoire une ville historique du Ferghana, certainement au détriment de l'application du critère national: Och, bien que majoritairement ouzbèke est située au Kirghizstan ; Khodjent est la capitale du nord du Tadjikistan. Symboliquement également, l'enclave de Chakhimardan est un territoire rattaché à l'Ouzbékistan parce qu'elle abrite un lieu de pèlerinage ouzbek ancien, les élites politiques soviétiques d'Asie centrale ayant intégré le fait religieux dans leurs critères de délimitation des territoires républicains.

Les frontières ainsi tracées devaient demeurer des limites internes à l'URSS et ne pas devenir des frontières internationales. Ce statut de frontière intra-soviétique garantissait une liberté de circulation et un franchissement aisé des limites administratives aux habitants de la vallée du Ferghana. Cette caractéristique justifie notamment le fait que le Ferghana soit demeurée une région trans-républicaine unie durant la période soviétique, ce qui explique pourquoi la complexité du tracé des frontières n'était alors qu'une question secondaire à l'échelle locale.

Depuis l'implosion de l'URSS, les frontières du Ferghana sont devenues des frontières internationales dans un contexte d'affirmation des États indépendants et de marquage des nouveaux territoires nationaux. Dans ce cadre, les premières difficultés de franchissement des frontières sont apparues en 1996 quand l'Ouzbékistan a compliqué le passage de sa frontière avec le Tadjikistan ${ }^{8}$. En outre, l'Ouzbékistan a été en 1998, le premier État présent au Ferghana à imposer un visa aux ressortissants des républiques voisines. Cependant, les Kirghizstanais riverains de la frontière bénéficient d'un régime allégé dès lors qu'ils ne sortent pas d'une bande frontalière d'une largeur de $100 \mathrm{~km}$ au maximum ${ }^{9}$. Par ailleurs, l'Ouzbékistan a équipé ses frontières depuis 1999 d'un système de fil de fer barbelés et de postes-frontières et a ainsi entravé et davantage contrôlé les déplacements intra-régionaux. L'évolution contemporaine du statut et de l'équipement des frontières du Ferghana rappelle que ce sont les politiques menées par les gouvernements qui font des frontières des lieux de difficultés et de tensions et non leur tracé.

\section{Les enclaves du Ferghana dans le contexte post-soviétique.}

Dans le contexte post-soviétique, les enclaves apparaissent comme des territoires d'autant plus originaux que les frontières héritées de l'URSS n'ont pas été remises en cause dans leur globalité ${ }^{10}$. Pourtant, un protocole d'accord avait été signé entre l'Ouzbékistan et le Kirghizstan en 2001 quant à l'établissement d'un corridor entre l'enclave de Sokh et le territoire ouzbékistanais en échange de la continuité territoriale entre Barak et le Kirghizstan $^{11}$. Mais, ce projet a été refusé par le Kirghizstan, à la suite de débats importants, notamment au parlement. Le sud du Ferghana conserve de ce fait toute sa complexité frontalière.

\footnotetext{
${ }^{8}$ Olimov, M., Olimova, S., «Xudjand : meždu Ferganoj i Tadžikistanoj » [Khodjent : entre le Ferghana et le Tadjikistan], Vestnik Evrazii - Acta eurasica, 1998, n¹-2 (4-5), pp. 203-220.

${ }^{9}$ Central Asia : Border Disputes and Conflict Potential, ICG Asia Report n ${ }^{\circ} 33,2002$, p. 13.

${ }^{10}$ L'Ouzbékistan mènerait cependant une politique expansionniste en intégrant arbitrairement des terroirs et des villages à son territoire pendant l'équipement de la frontière.

${ }^{11}$ Central Asia ..., p. 15.
} 
L'enclave kirghizstanaise de Barak est rattachée au district de Kara-Suu dans l'oblast' d'Och. Située à $4 \mathrm{~km}$ au nord de la frontière Kirghizstan-Ouzbékistan, l'enclave comprend une population légèrement supérieure à 600 habitants qui subit largement les conséquences de sa «situation d'anomalie territoriale $»^{12}$. Les problèmes concernent la circulation à destination et en provenance du Kirghizstan. Ils se manifestent tout particulièrement dans les secteurs de la santé et de la scolarisation des jeunes dès lors qu'ils fréquentent les grandes classes en raison l'absence d'infrastructures suffisantes au sein de l'enclave. Les politiques et la situation territoriales aboutissent à une accentuation de la dégradation du niveau de vie, déjà médiocre dans le contexte de la transformation post-soviétique. Cependant, si l'enclavement constitue une réalité contraignante, il demeure circonscrit à une population aux effectifs relativement réduits, celle de l'enclave.

Plus vastes, les enclaves ouzbékistanaises de Sokh et de Chakhimardan sont respectivement situées dans les parties amont du Sokh et de l'Ak-Suu, dans un environnement d'avant-chaînes et de montagnes. Sokh présente la particularité d'être l'enclave ferghanienne la plus peuplée. La population de cette vieille oasis tadjike dépassait en effet 42000 habitants en 1993 pour un territoire de $325 \mathrm{~km}^{2}{ }^{13}$. La délimitation des frontières de l'enclave avec la Kirghizie n'est pas encore nettement établie même si l'Ouzbékistan avait miné celles-ci pour faire face aux incursions du Mouvement islamiste d'Ouzbékistan (MIO) en 1999 et 2000. Depuis, les vives réactions des autorités kirghizstanaises locales ont cependant abouti au déminage partiel de cette frontière sensible alors même que le danger islamiste est désormais plus réduit ${ }^{14}$. Les relations entre ces deux enclaves et le territoire ouzbékistanais demeurent assez importantes et ne semblent guère souffrir de la traversée du territoire kirghizstanais, les gardes-frontières du Kirghizstan adoptant une attitude tolérante, à l'image de la politique gouvernementale ${ }^{15}$. Ainsi les véhicules ouzbékistanais circulent-ils sans difficulté sur le réseau kirghizstanais; des autocars réguliers relient Sokh à Kokand notamment et Chakhimardan accueille toujours des touristes et des pèlerins venus d'Ouzbékistan. La circulation longitudinale, aval-amont, Ouzbékistan-Ouzbékistan reste très largement supérieure à la circulation de piémont entre les différents secteurs kirghizstanaises car cette dernière subit davantage les contraintes du tracé des frontières et des politiques postsoviétiques, en particulier ouzbékistanaises. En effet, l'impossibilité pour les Kirghizstanais d'emprunter la route de plaine située en Ouzbékistan, impose à ces derniers de changer d'itinéraire au profit d'une ancienne route secondaire qui traverse les avant-chaînes kirghizstanaises. Mais cet axe qui relie la région de Batken aux autres régions du Kirghizstan traverse l'enclave de Sokh sur quelques kilomètres. L'enclave ouzbékistanaise contrôle de plus l'accès à la haute vallée du Sokh et de ses affluents où sont toujours situés quelques villages kirghizstanais malgré les politiques soviétiques de regroupement dans la plaine de cette population montagnarde ${ }^{16}$. Les postes-frontières et les contrôles tatillons des gardesfrontières en particulier ouzbékistanais constituent des entraves à la circulation au sein du territoire kirghizstanais, même si un flux régulier de véhicules existe, notamment un autocar Batken-Och. L'absence de continuité du réseau routier kirghizstanais signifie donc l'enclavement qui caractérise tout le sud-ouest du Kirghizstan. Compte tenu du dessin des

\footnotetext{
${ }^{12}$ Steck, B., Enclavement..., p. 130.

${ }^{13}$ Balland, D., «Diviser l'indivisible : les frontières introuvables des États centrasiatiques », Hérodote, 1997, n० 84, p. 97.

${ }^{14}$ Le chef historique du MIO, Juma Namangani a été tué au cours de combats en Afghanistan à l'automne 2001.

${ }^{15}$ Observations faites sur le terrain, août 2002.

${ }^{16}$ Cariou, A., Le jardin saccagé -Anciennes oasis et nouvelles campagnes d'Ouzbékistan, thèse de doctorat, université de Paris IV, 2002, pp. 262-263.
} 
frontières, les politiques des gouvernements indépendants produisent des contraintes de déplacement qui se révèlent plus difficile à supporter pour les populations kirghizstanaises que pour les populations ouzbékistanaises. La continuité territoriale kirghizstanaise ne garantit pas l'accessibilité de toutes les régions. Cette situation paradoxale souligne l'importance de la composante réticulaire de l'enclavement de même que la nature de la politique ouzbékistanaise.

Plus à l'ouest, dans la haute vallée de l'Isfara, est située l'enclave tadjikistanaise de Voroukh qui est rattachée à l'oblast' de Sogd (ancien oblast' de Leninabad). Très proche du territoire tadjikistanais, elle contient une population largement tadjike qui justifie l'existence de cette particularité territoriale. Mais si le dessin des frontières fait de ce territoire une enclave, le réseau routier l'intègre pleinement dans l'espace tadjikistanais ${ }^{17}$. Aucun marquage ne signale l'existence d'une frontière internationale entre le village de Voroukh et la ville d'Isfara. Aucun poste-frontière kirghizstanais ne gêne la circulation amont-aval dans cette vallée tadjike, d'autant plus que cette route ne dispose pas de connexion avec le réseau routier kirghizstanais. Il existe donc une continuité du réseau routier tadjikistanais, rendue possible grâce à l'attitude souple du gouvernement kirghizstanais. Paradoxalement, l'existence d'une enclave ne produit pas d'enclavement pour sa population résidente, à l'échelle locale. L'enclave de Voroukh est un fait cartographique, elle n'est pas une réalité géographique. Par contre, l'organisation du relief et le tracé des frontières imposent une rupture dans le réseau de transport terrestre kirghizstanais à l'ouest de Batken. En effet, la continuité territoriale kirghizstanaise n'est assurée que par des espaces montagneux où aucune voie de communication moderne n'existe. Dans ces conditions, la traversée du territoire tadjikistanais via Isfara est impérative pour accéder au district de Liaïliak et à l'ouest du district de Batken. De très nombreux postes de contrôle jalonnent la route transversale de piémont sur le territoire tadjikistanais, n'empêchant pas néanmoins la circulation de véhicules kirghizstanais ${ }^{18}$. L'extrémité occidentale de la partie kirghizstanaise du Ferghana connaît de ce fait une situation exceptionnelle d'enclavement double puisqu'elle n'est accessible que par la traversée de portions de territoire ouzbékistanais et tadjikistanais.

Le pourtour méridional du Ferghana, célèbre pour ses enclaves tadjikistanaises et ouzbékistanaises en Kirghizie, est donc une région où l'enclavement est une réalité postsoviétique marquante. Paradoxalement, alors que son territoire national est uni, le Kirghizstan est l'État qui semble le plus subir les évolutions politiques contemporaines tant l'enclavement y est généralisé, en particulier dans l'ouest de l'oblast' de Batken. Cette situation est de plus renforcée par le fait que, juste à l'ouest de Kyzyl-Kia, entre Markaz et Kadamjaï, la route Och-Batken est située en territoire ouzbékistanais, au pied des premiers contreforts du TianChan méridional ${ }^{19}$. Cependant, l'absence de connexion avec le réseau routier ouzbékistanais permet une libre circulation des véhicules et donne à cet axe un statut d'extraterritorialité. L'enclavement des régions kirghizstanaises demeure ainsi relativement souple, plus contraignant qu'empêchant, car le franchissement des frontières sur la route transversale de piémont reste autorisé et possible. L'évolution du système spatial dans le sud du Ferghana illustre combien la qualité des réseaux de transport détermine l'accessibilité des lieux et constitue, autant que le maillage territorial, une cause de l'enclavement ou du désenclavement.

\footnotetext{
${ }^{17}$ Observations faites sur le terrain, juin 2001.

${ }^{18}$ Cinq postes de contrôle en territoire tadjikistanais sont situés sur la route Batken-Isfara en plus du postefrontière.

${ }^{19}$ Observations faites sur le terrain, août 2002.
} 


\section{L'enclavement des Ferghana, un phénomène post-soviétique.}

À l'échelle du Ferghana dans son ensemble, le changement de statut des frontières et leur fermeture au moins partielle constituent des transformations majeures de la période postsoviétique. Associées aux conséquences de la guerre civile au Tadjikistan, ces mutations aboutissent à la rupture de la continuité spatiale régionale maintenue durant la période soviétique. En conséquence, un enclavement fort se manifeste, d'autant plus que l'on constate dans le même temps une baisse générale de la mobilité des populations en raison de la crise économique et sociale post-soviétique.

La fermeture des frontières inaugurée par l'Ouzbékistan entraîne la fragmentation de l'espace ferghanien. Sur la base des territoires nationaux indépendants, elle remet en cause l'unité du bassin qui fut une région et les relations ancestrales entre les plaines, les piémonts et la montagne fondées sur la complémentarité des espaces, tout comme l'organisation économique moderne mise au point par les Soviétiques. Les troupeaux ouzbékistanais, qui estivaient dans les montagnes du Kirghizstan, ne transhument plus ${ }^{20}$. La répartition de l'eau entre les républiques régulée largement par des barrages situés au Kirghizstan est devenue problématique et entache les relations internationales. Les flux traditionnels des produits alimentaires subissent également l'établissement de frontières rigides car il existe des interdictions de commercer et parce que les frontières sont devenues des lieux de rupture de charge. Sur le réseau routier ouzbékistanais en particulier, la circulation des véhicules immatriculés dans un État voisin est un fait rare qui nécessite une autorisation particulière liée notamment au paiement d'une taxe. Ainsi, les liaisons par autocar entre les principales villes de la région ont-elles été suspendues dès lors qu'elles intéressaient des localités de pays différents. Les difficultés de franchissement des frontières sont d'autant plus contraignantes et humiliantes pour la population du Ferghana que de nombreuses familles sont disséminées dans plusieurs républiques, alors même que les frontières divisent une population souvent homogène. Rendre visite à des parents proches ou retourner dans son kichlak ${ }^{21}$ d'origine nécessite fréquemment de se soumettre à l'autorité des gardes-frontières et à la vindicte de la milice. Ces difficultés de circulation soulignent la dégradation des conditions de vie et développent la dimension psychologique de l'enclavement chez les habitants du Ferghana affectés directement par cette politique de fermeture et de contrôle des frontières. L'instauration de limites administratives difficilement franchissables conduit au développement, dans le Ferghana, de régions distinctes en fonction de leur appartenance territoriale nationale, d'autant plus que les États connaissent des trajectoires de transition variées. Cependant, l'équipement des frontières ouzbékistanaises - la frontière OuzbékistanTadjikistan a même été minée - n'empêche pas l'existence de flux piétonniers et routiers non officiels mais tolérés par les gardes-frontières et les autorités.

Dans le contexte de la désintégration du système soviétique de production, la fermeture des frontières impose, d'autre part, une réorientation des flux au sein des territoires nationaux ${ }^{22}$. Volontaire ou contrainte, cette mutation soulève la question des relations qu'entretiennent chacun des Ferghana avec le reste du territoire dont il dépend. Le dessin des

\footnotetext{
${ }^{20}$ Observation faites sur le terrain, printemps 2001, été 2002.

${ }^{21}$ Désignant initialement le pâturage d'hiver dans le monde turco-iranien, le terme kichlak signifie par extension le village en Asie centrale « sédentaire ».

${ }^{22}$ Thorez, J., «Montagnes et espaces nationaux post-soviétiques en Asie centrale (Kirghizstan, Ouzbékistan, Tadjikistan) », à paraître en 2003 dans les actes du colloque «Traverser les montagnes » organisé par l'université de Savoie en 2002.
} 
frontières et l'organisation du relief rendent nécessaire la traversée d'axes montagneux pour relier les Ferghana avec Tachkent, Bichkek et Douchanbe, les capitales nationales. Dans le cadre post-soviétique, l'inadaptation des réseaux de transport au territoire national héritée de la période soviétique et l'énergie du relief ${ }^{23}$ entravent la circulation, en particulier en saison hivernale, malgré des aménagements récents. Suivant les pays, cette situation conduit à un développement plus ou moins important de l'enclavement, en fonction de la qualité des réseaux de transport, des moyens financiers et techniques mis en œuvre pour adapter l'espace au territoire national et des contraintes naturelles (voir carte : «l'enclavement dans le Ferghana post-soviétique »). La route Kokand-Tachkent emprunte le col de Kamtchik d'altitude moyennement élevée (2232 m), alors que les routes Khodjent-Douchanbe et OchBichkek traversent chacune plusieurs axes montagneux et passent respectivement aux cols de Chakhristan (3359 m) et d'Anzob (3370 m) au Tadjikistan, et aux cols de Tyoo-Achuu (3586 m) et d'Alabel' (3186 m) au Kirghizstan. Si la route ouzbékistanaise reste ouverte quasiment toute l'année, en particulier grâce au percement de deux tunnels sous le col, la circulation entre le Ferghana et Bichkek demeure ralentie et incertaine l'hiver, malgré d'importants investissements pour l'amélioration de la chaussée et l'existence d'un tunnel sous le col de Tyoo-Achuu. Au Tadjikistan, les cols d'Anzob et de Chakhristan sont fermés de novembre à avril de sorte que la région de Khodjent est isolée du reste du territoire national en hiver. Enfermé dans ses nouvelles frontières au nord, le Ferghana tadjikistanais endure alors un enclavement saisonnier fort, très préjudiciable au développement économique et social, supérieur à l'isolement relatif que connaît le Ferghana kirghizstanais. Plus proche de la capitale et mieux relié à celle-ci, le Ferghana ouzbékistanais ne connaît quant à lui qu'un enclavement limité.

L'enclavement affecte à des degrés variés toute la région du Ferghana. Produit des politiques post-soviétiques, il est subi par les habitants, qui voient leurs pratiques spatiales évoluer rapidement, en particulier au Tadjikistan,. Le transport aérien permet néanmoins de s'affranchir partiellement de cette situation. Mais, nouveauté post-soviétique, les tarifs élevés associés au processus de paupérisation de la majorité de la population conduisent la majorité des habitants à négliger ce mode de transport alors même que les réseaux domestiques se sont rétractés, en particulier au Kirghizstan. La situation face à l'enclavement apparaît dès lors comme un critère de différenciation sociale dans un contexte de renforcement des inégalités.

\section{Conclusion}

Le cas du Ferghana constitue un bel exemple d'enclavement emboîté tant chacune des échelles - internationale, nationale, locale - illustre ce phénomène. Changement intervenu dans la période contemporaine, l'enclavement transforme le quotidien des habitants et des sociétés et participe pleinement de la crise post-soviétique. Ce processus de fermeture et de repli bouleverse le système spatial : alors que l'URSS avait maintenu et renforcé l'unité régionale du Ferghana, dix ans d'indépendance aboutissent à la fragmentation de l'espace régional, dans le cadre de l'affirmation des territoires et des États nationaux post-soviétiques, et à l'émergence de trois Ferghana nationaux distincts: le Ferghana kirghizstanais, le Ferghana ouzbékistanais et le Ferghana tadjikistanais. Par ailleurs, le Ferghana était intégré au sein de l'Asie centrale, ainsi que dans l'URSS. Désormais, ses fragments se trouvent en situation d'isolement au cœur du continent asiatique. L'ampleur des transformations n'interdit

\footnotetext{
${ }^{23}$ L'altitude moyenne des chaînes qui encadrent le Ferghana dépasse souvent $4000 \mathrm{~m}$.
} 
cependant pas de s'interroger sur le degré de résilience du système spatial ferghanien ${ }^{24}$, tant la période écoulée depuis l'implosion de l'URSS est courte, la situation encore très incertaine et l'inertie des structures et des pratiques spatiales importante.

\footnotetext{
${ }^{24}$ Aschan-Leygonie, C., «Vers une analyse de la résilience des systèmes spatiaux », L'Espace géographique, tome $29, \mathrm{n}^{\circ} 1,2000$, pp. 64-77.
} 